dence of clinically significant outcomes as a result of this remains low. Massive carbon dioxide embolism causing sudden cardiac arrest is highly unusual. Nevertheless, all cardiac surgical teams should be aware of such unlikely events.

Alan P. Kypson, MD, FACS Division of Cardiothoracic Surgery The Brody School of Medicine East Carolina University Greenville, NC 27834 doi:10.1016/j.jtcvs.2006.09.001

\section{Are absorbable sutures inadequate to close the sternum?} To the Editor:

We read with interest the article by Usui and colleagues. ${ }^{1}$ The authors conclude that polydioxanone cord is not safe to close the sternum. We have tried to address a few relevant issues on this matter.

The mechanical performance of synthetic absorbable sutures is similar to that of stainless steel. ${ }^{2}$ Nonetheless, clinical use of absorbable sutures to close the sternum has not been widely reported. In 1994, we published a series of 216 consecutive sternotomies using size 1 polyglyconate monofilament sutures (Maxon, Davis and Geck) to close the sternum, ${ }^{3}$ and in the last 12 years, we adopted the same technique in every patient undergoing median sternotomy, hemiclamshell approach, or transmanubrial approach. No cases of sternal dehiscence were observed in more than 400 consecutive patients, even in the case of resternotomy.

Preoperative risk factors for sternal dehiscence include use of thoracic grafts, diabetes mellitus, chronic obstructive pulmonary disease, obesity, and smoking. ${ }^{2}$ All patients that had broken sutures in the report by Usui and colleagues ${ }^{1}$ presented with at least one such risk factor. However, these risk factors usually lead to late complications of sternal wound healing, whereas all 3 events in this report occurred within 2 weeks postoperatively, and macroscopic findings of the broken sutures suggest that failure of polydioxanone cord might be caused by abrasion injury as a result of friction with the bone edges. In fact, early sternal disruptions shortly after surgical intervention are more likely to be related to an inappropriate closure technique or suboptimal use of suture materials. In addition, faulty sternal splitting or paramedian sternotomy can lead to improper approximation of the sternum, the uneven margins of which can injure the sutures.

Usui and colleagues ${ }^{1}$ did not describe how polydioxanone cord was used to close the sternum. After median sternotomy, we routinely apply only one transsternal stitch of polyglyconate to approximate the manubrium and 2 to 3 double-loop stitches that are passed through the parasternal intercostal spaces, ensuring a double ring for each space. By using this method, the suture strength and sternal stability are enhanced. Furthermore, the absorbable suture should be handled with care because the contact with surgical instruments to clamp the knot or hold the suture in place can seriously damage the monofilament and should be avoided in any circumstance.

Polydioxanone and polyglyconate are similar in performance with respect to bursting strength. ${ }^{3}$ Both have high tensile strength, minimal tissue reaction, good memory, and elasticity (which are better than steel wire and nylon). However, negative results have been reported only with polydioxanone, and this might indicate a different clinical performance of the 2 materials with respect to sternal closure. ${ }^{1,4}$

One has to consider, however, that dehiscence of a median sternotomy after cardiothoracic surgery is about $1 \%$ in the literature. ${ }^{2}$ Therefore the figure of $0.8 \%$ (3/ 350 ) in the report by Usui and colleagues ${ }^{1}$ should not be thought of as unacceptable.

In conclusion, our long-lasting experience has proved that size 1 polyglyconate monofilament sutures are safe and effective for sternal closure in noncardiac surgery, and we are convinced that monofilament absorbable sutures are at least as good as steel wire to close the sternum after complete or partial sternotomy.

$$
\begin{array}{r}
\text { Xue Ning Yang, MD } \\
\text { Ugo Pastorino, MD } \\
\text { Division of Thoracic Surgery } \\
\text { Istituto Nazionale Tumori } \\
\text { Via Venezian 1, } 20133 \\
\text { Milan, Italy }
\end{array}
$$

E-mail: ugo.pastorino@istitutotumori.mi.it

\section{References}

1. Usui A, Oshima H, Akita T, Ueda Y. Polydioxane (PDS) cord has insufficient reliability to securely close the sternum. $J$ Thorac Cardiovasc Surg. 2006;131:1174-5.

2. Losanoff JE, Jones JW, Richman BW. Primary closure of median sternotomy: tech- niques and principles. Cardiovasc Surg. 2002; 10:102-10.

3. Pastorino U, Muscolino G, Valente M, et al. Safety of absorbable suture for sternal closure after pulmonary or mediastinal resection. J Thorac Cardiovasc Surg. 1994;107:596-9.

4. Mulch J, Stertmann WA, Kling D, Scheld $\mathrm{HH}$. Closure of longitudinal sternotomy with absorbable sutures. Thorac Cardiovasc Surg. 1986;34:191-3.

doi:10.1016/j.jtcvs.2006.08.024

\section{Bonnano's catheter: A less invasive and cost-effective alternative for drainage of pleural effusion To the Editor:}

We read with interest the article by Chetty and colleagues. ${ }^{1}$ Bonnano's catheter has been proposed as a less invasive and costeffective alternative for drainage of a pleural effusion. We congratulate them for their cost-effective and innovative approach of using a suprapubic catheter to drain the pleura.

Pigtail catheters using the modified Seldinger technique have been found to be well tolerated by the patient and are highly effective in drainage of pleural serous and chylous effusions but less efficacious in drainage of hemothorax or pneumothorax and empyema with acceptable risk of complications. $^{2}$

We agree that akin to small catheters using the Seldinger technique,, 3 Bonnano's catheter can be used. However, with the length of the trocar and the mounted catheter being approximately $30 \mathrm{~cm}$, one can lose track of the distance traveled in the thoracic cavity because there are no obvious markings on the catheter itself, and one can injure vital organs in the chest cavity, as in our institution's experience (unpublished report), in which a misplaced Bonnano's catheter pierced the left ventricle, and the tip of the catheter was found in the aortic root.

Before introduction of Bonnano's catheter into general medical and surgical use to drain the pleura, this technique needs to be validated, and the results of a prospective trial are awaited. We suggest that modifications to the catheter should include surface markings, thereby allowing the distance traveled to be gauged, and reduction of the length of the trocar, thereby reducing the risk of untoward complications.

These catheters might be useful for drainage of simple pleural effusions after surgical intervention. Complications might be related to the misplacement of the trocar because 